\title{
Growth and magnetic characterization of Co nanoparticles obtained by femtosecond pulsed laser deposition
}

\author{
A. Cebollada \\ Instituto de Microelectrónica de Madrid (IMM), Isaac Newton 8, 28760 Tres Cantos, Spain and FOCUS Center, \\ University of Michigan, 450 Church St., Ann Arbor, Michigan 48109, USA
}

J. M. García Martín and C. Clavero

Instituto de Microelectrónica de Madrid (IMM), Isaac Newton 8, 28760 Tres Cantos, Spain

L1. Balcells

ICMAB-CSIC, Campus UAB, 08193 Bellaterra, Barcelona, Spain

S. Estradé, J. Arbiol, and F. Peiró

Departamento de Electrónica, Ingeniería y Materiales Electrónicos (EME), Universidad de Barcelona, C/Martí i Franques 1, 08028 Barcelona, Spain

C. Smith and R. Clarke

Department of Physics and FOCUS Center, University of Michigan, 450 Church St., Ann Arbor, Michigan 48109, USA

L. Martínez, Y. Huttel, and E. Román

Instituto de Ciencia de Materiales de Madrid (ICMM-CSIC), 28029 Cantoblanco, Madrid, Spain

N. D. Telling

Magnetic Spectroscopy Group, Daresbury Laboratory, Warrington WA4 4AD, United Kingdom and School of Earth, Atmospheric and Environmental Science, University of Manchester, Oxford Road, Manchester M13 9PL, United Kingdom

G. van der Laan

Magnetic Spectroscopy Group, Daresbury Laboratory, Warrington WA4 4AD, United Kingdom and

Diamond Light Source Ltd., Chilton, Didcot, Oxfordshire OX11 ODE, United Kingdom

(Received 10 February 2008; revised manuscript received 1 December 2008; published 12 January 2009)

\begin{abstract}
We present a detailed study on the morphology and magnetic properties of Co nanostructures deposited onto oxidized Si substrates by femtosecond pulsed laser deposition. Generally, Co disks of nanometric dimensions are obtained just above the ablation threshold, with a size distribution characterized by an increasingly larger number of disks as their size diminishes, and with a maximum disk size that depends on the laser power density. In $\mathrm{Au} / \mathrm{Co} / \mathrm{Au}$ structures, in-plane magnetic anisotropy is observed in all cases, with no indication of superparamagnetism regardless of the amount of material or the laser power density. Magnetic force microscopy observations show coexistence of single-domain and vortex states for the magnetic domain structure of the disks. Superconducting quantum interference device magnetometry and x-ray magnetic circular dichroism measurements point to saturation magnetization values lower than the bulk, probably due to partial oxidation of the Co resulting from incomplete coverage by the $\mathrm{Au}$ capping layer.
\end{abstract}

DOI: 10.1103/PhysRevB.79.014414

PACS number(s): 75.75.+a

\section{INTRODUCTION}

Pulsed-laser deposition (PLD) is a versatile technique for the deposition of ultrathin films of practically any material. Recently, the use of femtosecond (fs) lasers for PLD (fsPLD) has become an active field of research from several different aspects. The use of ultrafast lasers for ablation of materials has principally two distinctive characteristics with respect to nanosecond (ns) PLD: first, fs lasers do not interact with the ejected material since the pulse has finished well before any ablation process of the target takes place. This enables studies of the fundamental thermodynamics and hydrodynamics of particle formation. Second, short laser pulses heat up the material to much higher temperatures than nano- second pulses with comparable fluence because the energy is delivered before any significant thermal conduction into the target's bulk can occur.

From a theoretical point of view, different approaches have tried to identify the physical process for ablation of material from a solid target upon irradiation with fs pulses. In particular, they point to different mechanisms such as liquid phase ejection and fragmentation, homogeneous nucleation and decomposition, and spinodal decomposition. ${ }^{1-5}$ In addition, several theoretical and experimental approaches have also revealed some insight into the ultrafast laser-pulsetarget interaction. This is of particular interest for understanding the incident laser's effects on the target morphol- 
ogy, the resulting plasma, and the nature of the species ejected from the target. ${ }^{6-15}$

In spite of this effort, however, very little has been done on the nature and properties of the material obtained from this ablation process once it is deposited on a substrate surface, either in the form of a continuous film or as nanoparticles. Deposition of continuous thin films using fs lasers has been successful in the case of oxides or nitrides, ${ }^{16-21}$ with ablation in the presence of a gas in the deposition chamber that reacts or interacts to some extent with the ejected material, leading to the growth of a continuous film on the substrate.

On the other hand, several groups have recently started to study the deposition and physical properties of nanoparticles obtained by fs-PLD from a variety of targets, such as Ge, ${ }^{22}$ Permalloy, ${ }^{23} \mathrm{Si}^{24,25} \mathrm{Ti}^{26}{ }^{26} \mathrm{Al},{ }^{27} \mathrm{Ni},{ }^{28,29}$ or $\mathrm{Fe} .{ }^{30}$ The common feature is that different materials seem to behave roughly the same when irradiated with intensities around the threshold of plasma formation, typically $10^{12}-10^{13} \mathrm{~W} / \mathrm{cm}^{2}$, leading to nanoparticle formation, with radii of tens of $\mathrm{nm} .{ }^{31}$ Most theoretical calculations $s^{2,4,5,10}$ attribute the generation of these particles to the ejection of clusters of material of different sizes down to individual atom dimensions. Experimentally, several studies have reported a maximum in the particle size distribution at some specific particle size, with a mean particle radius between 5 and $25 \mathrm{~nm}$ depending on the material. As we will show below a great deal of care is necessary in characterizing the size distribution at these small dimensions in order to avoid artifacts due to the resolution limit of the particular method that is used to determine the particle size. Another important issue is the actual shape of the deposited clusters of material. They are usually described as particles that one intuitively identifies with more or less spherical entities. However, if the cluster arrives at the substrate in a liquid state, it is likely to become deformed, flattening down to a disklike shape after making contact with the substrate. This geometry could also result from plastic deformation of solid particles impinging at very high ballistic velocities.

For all these reasons, fs-PLD is an interesting emergent technique for the fabrication of magnetic nanostructures with a wide size range (that could lead to a controlled monodisperse size if, for example, a quadrupolar mass filter is attached in the deposition chamber) and exhibiting interesting geometries. However, very few works related to this matter have been published so far. Reilly et al. ${ }^{23}$ compared the morphology of $\mathrm{Ni}_{80} \mathrm{Fe}_{20}$ thin films using a high-average-power free-electron laser (650 fs pulse width) and an amplified Ti:sapphire system (150 fs pulse width), studying the different morphologies, crystalline nature, and magnetization curves. Ausanio et al..$^{28}$ and Amoruso et al. ${ }^{30}$ also reported the synthesis, structure, morphology, and magnetic characterization of $\mathrm{Ni}$ and $\mathrm{Fe}$ nanoparticles or, as they call them, nanocrystal films. Very recently, Liu et al. ${ }^{29}$ reported also on the generation of Ni nanoparticles by ultrafast PLD, but no magnetic characterization was included. In addition, the influence of surface-plasmon resonances in the magneto-optical (magnetoplasmonic) activity of noble-metal/ferromagnet/noblemetal continuous films and nanodiscs has recently been highlighted, ${ }^{32-35}$ and there is a growing interest in exploring similar phenomena in systems fabricated using innovative methods.
Considering the recent interest in novel experimental techniques to obtain nanostructured magnetic materials, we have carried out a complete study focusing our attention on the fabrication and characterization of Co nanoparticles obtained by fs-PLD. Here we report on a comprehensive investigation of the fabrication, size distribution, morphology, structure, and magnetic properties of Co nanoparticles deposited by fs-PLD.

\section{EXPERIMENTAL APPROACH AND SAMPLE DEPOSITION}

PLD was carried out in an ultrahigh-vacuum (UHV) system previously described ${ }^{22}$ using a Ti:sapphire laser (Clark MXR CPA-2001) with a pulse width of 120 fs, a wavelength of $780 \mathrm{~nm}$, an energy per pulse of $800 \mu \mathrm{J}$, and repetition rates of $1 \mathrm{kHz}$ or $125 \mathrm{~Hz}$. A $45^{\circ}$ incidence angle was maintained, with $p$ polarization. The laser was focused down on the target surface achieving area power densities which ranged between $2 \times 10^{12}$ and $3.3 \times 10^{13} \mathrm{~W} / \mathrm{cm}^{2}$. The target to substrate distance was $5 \mathrm{~cm}$ and the incident-beam power was varied using an attenuator. The ablation target manipulator can hold up to four different $1 \mathrm{in}$. targets with full individual rotation and selective clocking motion. Rotation and rastering allow for uniform removal of target material with successive laser shots, avoiding damage of the target by the beam. ${ }^{22}$ Two different sets of samples, with 1 min Co deposition for all samples, were grown on Si substrates. The first set consisted of Co deposits grown with different laser powers for morphological studies with atomic force microscopy (AFM) and scanning electron microscopy (SEM). A second set consisted of $\mathrm{Au} / \mathrm{Co} / \mathrm{Au}$ trilayers deposited with different laser powers and deposition times for Co. This second set of samples was used for compositional [by energydispersive x-ray spectroscopy (EDS)], structural [by transmission electron microscopy (TEM)], and magnetic characterization. Au was evaporated from an effusion cell with the evaporation rate calibrated by determining the thickness of Au layers from x-ray reflectometry. The thickness of the Au buffer and capping layers was 16 and $6 \mathrm{~nm}$, respectively. Such capping layer thickness was thin enough to be able to extract information from the material underneath by means of x-ray magnetic circular dichroism (XMCD) and had proven to be effective in protecting Co layers and nanodisc layers against oxidation in previous studies where the samples had been prepared by means of MBE, sputtering, and lithographic processes..$^{34,35}$

$\mathrm{Si}$ substrates were cut into $1 \times 1 \mathrm{~cm}^{2}$ squared pieces, cleaned ultrasonically in baths of trichloroethylene, acetone, and methanol, blow dried with nitrogen, and loaded into the deposition chamber. Prior to the growth of the different layers at room temperature (RT), the Si substrates were outgassed at $600{ }^{\circ} \mathrm{C}$ for $45 \mathrm{~min}$. AFM and magnetic force microscopy (MFM) images were taken at ambient conditions using a Nanotec ${ }^{\mathrm{TM}}$ microscope operating in a noncontact dynamic mode. For MFM, the tip was scanned at a constant lift height above the sample, typically $50 \mathrm{~nm}$, and the phase shift, proportional to the force gradient, was measured. Silicon probes from Nanosensors ${ }^{\mathrm{TM}}$ with a cantilever force con- 
stant of $\sim 3 \mathrm{~N} / \mathrm{m}$, resonant frequency of $\sim 70 \mathrm{kHz}$, and pyramidal tip with radius below $10 \mathrm{~nm}$, were used. For MFM, the tips were coated with $\mathrm{Cr} / \mathrm{Co} / \mathrm{Cr}$ trilayers by electron-beam (e-beam) thermal evaporation, with the thickness of Co being varied between 20 and $60 \mathrm{~nm}$ in order to have a suitable magnetic moment for each experiment: too low a magnetic moment would produce a poor signal-to-noise ratio, while a too high moment would alter the magnetic state of the nanoparticle being analyzed. All the tips were magnetized along the pyramid axis.

Structural and chemical characterization was carried out by high-resolution transmission electron microscopy (HRTEM) and electron energy-loss spectroscopy (EELS) in a Jeol 2010 FEG at $200 \mathrm{keV}$, with a GIF spectrometer. The composition of trilayers was determined by EDS. Highresolution morphology studies of the deposited nanoparticles were made in a field-emission SEM (FEI Nova Nanolab) with $3.5 \mathrm{~nm}$ resolution operating at $30 \mathrm{keV}$. Magnetization measurements were carried out by using an rf superconducting quantum interference device (SQUID) magnetometer from Quantum Design in applied fields of up to $20 \mathrm{kOe}$.

$\mathrm{X}$-ray photoemission spectroscopy (XPS) measurements have been performed in a separate UHV chamber with a base pressure of $1 \times 10^{-10}$ mbar. The angle between the hemispherical analyzer (Specs-PHOIBOS100) and the plane of the surface was kept at $60^{\circ}$ and used the $\mathrm{Mg} \mathrm{K \alpha}$ line (1253.6 $\mathrm{eV})$ for the $\mathrm{x}$-ray radiation. XPS spectra were recorded after removing a 15 - $\AA$-thick layer of materials from the samples by mild $\mathrm{Ar}^{+}$sputtering at $1 \mathrm{keV}$ with a known ion sputtering rate. ${ }^{36}$ The ion sputtering was performed in order to remove the contaminants from the surface and to enhance the photoemitted signal from the Co $2 p$ core levels. It should be noted that the removal of the surface layers of the sample is very appropriate for the further quantification of oxygen in the sample since it removes the oxygen atoms adsorbed from the air. The spectra were recorded with photon energy steps of 0.1 and $15 \mathrm{eV}$ pass energy. Prior to the data analysis, the contributions of the $\operatorname{Mg} K \alpha$ satellite lines were subtracted and the spectra were subjected to a Shirley-type background subtraction formalism.

The magnetic characterization of the samples using XMCD was performed by measuring the $\mathrm{x}$-ray-absorption spectra (XAS) in total electron-yield mode on beamline 5U.1 of the Synchrotron Radiation Source (SRS) at Daresbury, UK. All XMCD measurements were performed at room temperature using the portable octupole magnet system (POMS), which can reach magnetic fields up to $\pm 0.9 \mathrm{~T}$. With the POMS the magnetic field can be applied in any direction, although the results presented here were measured by applying the magnetic field along the incident x-ray beam. The direction of the applied magnetic field was reversed at each photon energy point of the spectrum. The degree of polarization was $53.4 \%$ as deduced from a reference sample with the configuration $30 \AA \mathrm{Al} / 40 \AA \mathrm{Co} / \mathrm{Al}_{2} \mathrm{O}_{3}(0001)$. Incomplete magnetization saturation was corrected with the values of the saturation magnetic field extracted from the Kerr hysteresis loops measured in polar geometry.

Due to the nanoparticle nature of the Co deposits, no direct determination of the deposition rate was possible, as, for example, with standard X-ray reflectivity (XRR) measure-

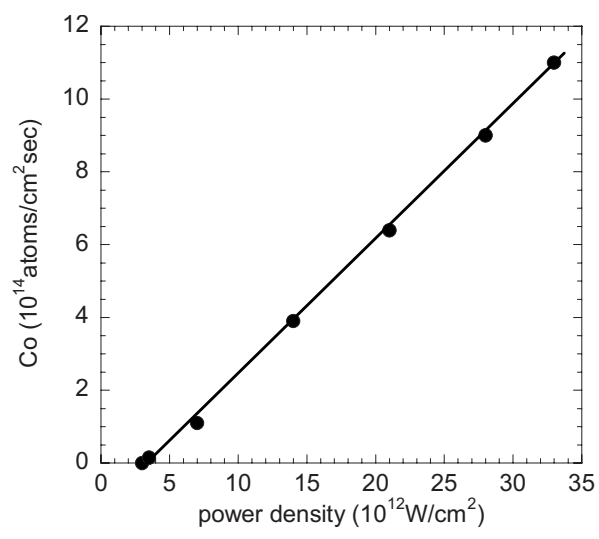

FIG. 1. Co deposition rate as a function of laser power density. A linear behavior is obtained, intersecting zero deposition at 3 $\times 10^{12} \mathrm{~W} / \mathrm{cm}^{2}$, in good agreement with the experimentally observed ablation threshold.

ments of a continuous layer. The Co deposition rate was indirectly determined by EDS measurements from $\mathrm{Au} / \mathrm{Co} /$ $\mathrm{Au} / \mathrm{glass}$ trilayers where the $\mathrm{Au}$ thickness was known. $\mathrm{Au}$ grows with (111) texture on glass as well as on Co (Ref. 37) and therefore it is possible to know the number of $\mathrm{Au}$ atoms per unit area. Since EDS measurements provide the relative number of $\mathrm{Co}$ atoms per $\mathrm{Au}$ atom, it is therefore possible to extract the number of Co atoms per unit area and as a consequence the Co deposition rate. In Fig. 1 we show the Co deposition rates obtained as a function of laser fluence. As can be seen, the rate exhibits a linear behavior, starting from a threshold at around $3 \times 10^{12} \mathrm{~W} / \mathrm{cm}^{2}$. This agrees very well with the experimentally observed minimum power needed to ablate material off the target (ablation threshold).

\section{CHARACTERIZATION}

\section{A. Morphology \\ 1. $\mathrm{Co} / \mathrm{Si}$}

To study the laser power dependence of the particle morphology and size distribution, Co was deposited onto oxidized Si substrates at different laser power densities ranging from $3.5 \times 10^{12}$ to $3.3 \times 10^{13} \mathrm{~W} / \mathrm{cm}^{2}$ with 1 min deposition time in all cases. The results are shown in Fig. 2, where SEM images are presented for representative powers. Ellipsoidal nanoparticles, close to circular and of different diameters, are observable in all cases, with a larger amount of nanoparticles for increasing power. For $3.5 \times 10^{12} \mathrm{~W} / \mathrm{cm}^{2}$, just above the ablation threshold, there is already a wide distribution of particle diameters from around $70 \mathrm{~nm}$ down to the resolution limit of the microscope. As expected, the number of particles increases with laser power, all the way up to the maximum power density of $3.3 \times 10^{13} \mathrm{~W} / \mathrm{cm}^{2}$. In some cases, and for all laser powers, an annular structure is observed, indicative of a disklike or ringlike geometry rather than spherically shaped particles. Coalescence, especially for high powers, and perimeter decoration with smaller particles are also observed.

In order to obtain the particle size distribution vs laser power, we have performed statistics from different SEM im- 

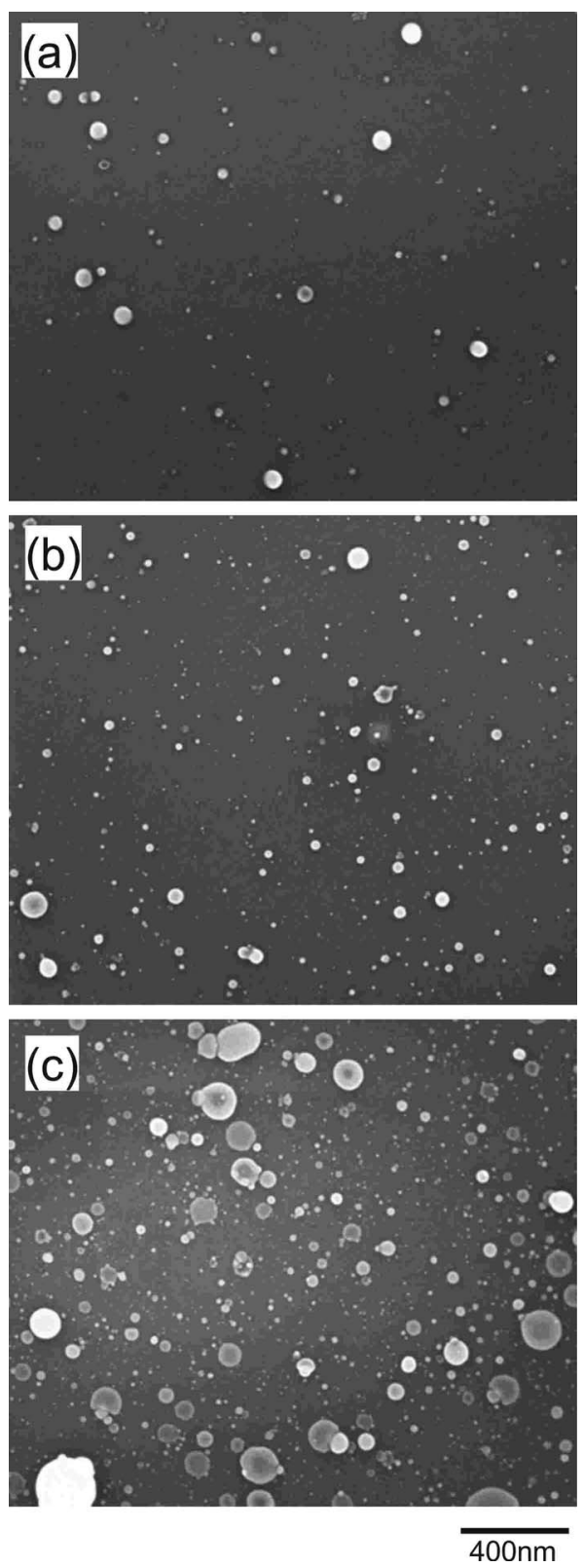

FIG. 2. SEM images obtained for 1 min depositions of Co on $\mathrm{Si}$ at (a) $3.5 \times 10^{12} \mathrm{~W} / \mathrm{cm}^{2}$, (b) $7 \times 10^{12} \mathrm{~W} / \mathrm{cm}^{2}$, and (c) 2.8 $\times 10^{13} \mathrm{~W} / \mathrm{cm}^{2}$.

ages for all the laser powers, measuring the in-plane diameter. For higher laser power density, the observed maximum disk size increases, as shown in Fig. 3; for instance, the largest in-plane diameter found for $2.8 \times 10^{13} \mathrm{~W} / \mathrm{cm}^{2}$ is 240 $\mathrm{nm}, 150 \mathrm{~nm}$ for $7 \times 10^{12} \mathrm{~W} / \mathrm{cm}^{2}$, and $120 \mathrm{~nm}$ for 3.5 $\times 10^{12} \mathrm{~W} / \mathrm{cm}^{2}$. We also performed a similar statistical on AFM images of selected samples, yielding similar results except for the small diameter range, where the convolution effect with the AFM tip radius $(\sim 10 \mathrm{~nm})$ affects the results. Although previous studies reported a characteristic particle diameter between 5 and $10 \mathrm{~nm},{ }^{13,24,25}$ our experimental data do not show a peak in the particle size distribution, but rather a continuous increase in the number of particles as their size is reduced until the resolution of the available images prohibits determining such statistics. Another factor playing a

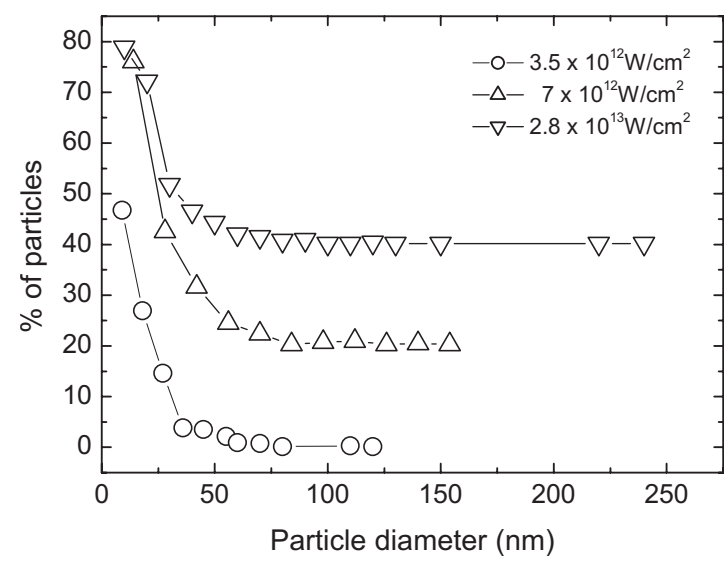

FIG. 3. In-plane particle diameter distribution (normalized to the total amount of computed particles) extracted from representative SEM images taken for $\mathrm{Co} / \mathrm{Si}$ samples deposited at three selected laser power densities. The distributions for $7 \times 10^{12}$ and 2.8 $\times 10^{13} \mathrm{~W} / \mathrm{cm}^{2}$ have been vertically displaced by $20 \%$ and $40 \%$, respectively, for clarity. For all densities, the number of particles increases gradually and continuously as the particle diameter is reduced. However, for higher power density larger nanostructures can be produced.

role could be the different optical, thermal, and rheological properties of the materials studied in this work compared with $\mathrm{Co}$, being therefore likely to produce slightly different particle size distributions.

Although the apparent morphology of the deposited particles in the SEM images is generally circular, some nanostructures appear as nanorings and nanorosaries as can be observed in Fig. 4. Since SEM is not very sensitive in depth, AFM images were taken in representative samples to investigate the actual shape of the deposited Co nanostructures. The AFM measurements revealed that the shape of the particles referred to as circular is actually oblate semispherical, with an aspect ratio (diameter of the base divided by the polar axis) between 3 and 15. The shape of the nanorings is similar to that of a volcano, whereas the nanorosaries are circular structures surrounded by small particles in the form of droplets. These findings indicate that the liquid state plays an important role in the formation of at least some of the nanostructures. One possibility is that Co clusters arrive in liquid state at the substrate and exhibit a plastic behavior after impinging on the surface, freezing into the finally observed morphology. Another interpretation is that Co clusters hit the substrate in a solid state, and then melt due to the energy delivered in the collision, turning back to a solid after thermalization with the substrate.

\section{2. $\mathrm{Au} / \mathrm{Co} / \mathrm{Au} / \mathrm{Si}$}

For further magnetic characterization, $\mathrm{Au} / \mathrm{Co} / \mathrm{Au}$ trilayered structures grown on $\mathrm{Si}$ and with 1 min deposition of $\mathrm{Co}$ at different power densities were used. AFM images for the different samples are shown in Fig. 5.

As can be observed, the Au layer does not completely cover the Si substrate, but deposits as Au aggregates, roughly $100 \mathrm{~nm}$ in lateral dimension and close to each other. For Co 

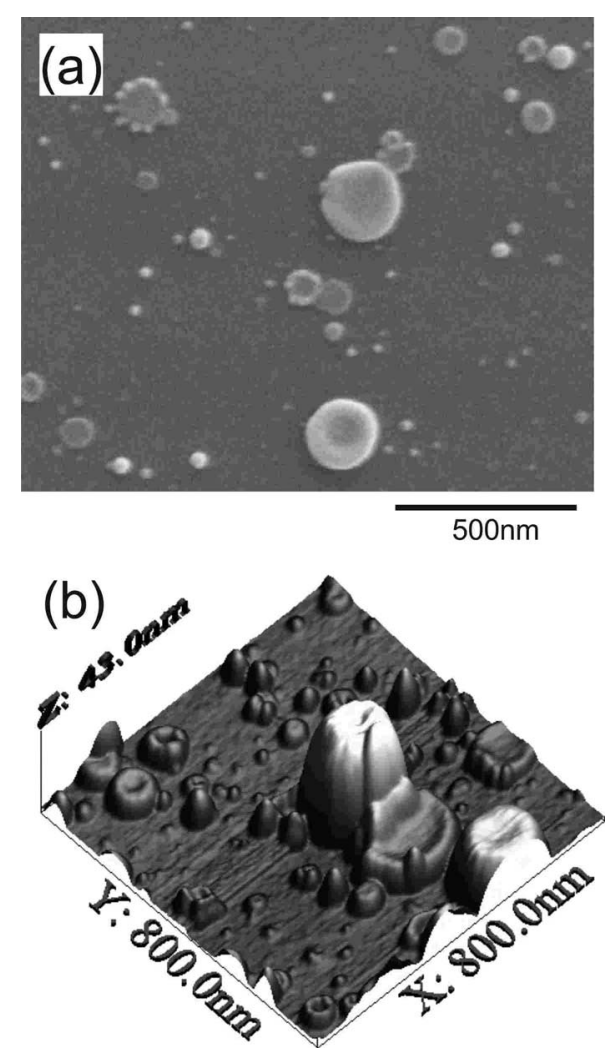

FIG. 4. Images for selected $\mathrm{Co} / \mathrm{Si}$ samples grown at 3.3 $\times 10^{13} \mathrm{~W} / \mathrm{cm}^{2}$ exhibiting shapes different from disks. Both nanorings and nanorosaries are observed. (a) SEM image. (b) AFM image.

deposited at $2 \times 10^{12} \mathrm{~W} / \mathrm{cm}^{2}$, just below the determined ablation threshold, the morphology is almost indistinguishable from the bare $\mathrm{Au}$ layer, implying a very small Co particle size or simply the absence of Co in the layer (keeping in mind the $3 \times 10^{12} \mathrm{~W} / \mathrm{cm}^{2}$ ablation threshold). No particle is observed by AFM and only one feature, which could be a Co particle, was observed in the SEM measurements. This indicates that the amount of Co ablated at this subthreshold laser power is very small.

At $3.5 \times 10^{12} \mathrm{~W} / \mathrm{cm}^{2}$ power density, a few features on top of the Au layer are observable, both by AFM and SEM. Diameters and heights vary, but range roughly between 50 and $200 \mathrm{~nm}$ in diameter and 10-20 nm in height. This implies that the particles are actually disks with an aspect ratio that might actually depend on the aggregate size. For example, the smaller Co particles are $50 \mathrm{~nm}$ in diameter and 20 $\mathrm{nm}$ high, while others, although a minority in number, have a distinctive ring shape, with a central dip that may or may not leave the central part of the ring covered with Co. This is more noticeable for $7 \times 10^{12} \mathrm{~W} / \mathrm{cm}^{2}$, where Co rings of two types, either with the central part covered or uncovered with $\mathrm{Co}$, are observed. For $1.4 \times 10^{13} \mathrm{~W} / \mathrm{cm}^{2}$, a larger population of Co particles is clearly observed, even though in this specific case no ringlike structures were detected. The same trend, with increasing amount of Co particles as a function of the laser power, is observed for the $3.3 \times 10^{13} \mathrm{~W} / \mathrm{cm}^{2}$ samples.

Finally, regarding the structural characterization, TEM analysis was carried out on selected $\mathrm{Au} / \mathrm{Co} / \mathrm{Au} / \mathrm{Si}$ samples, in
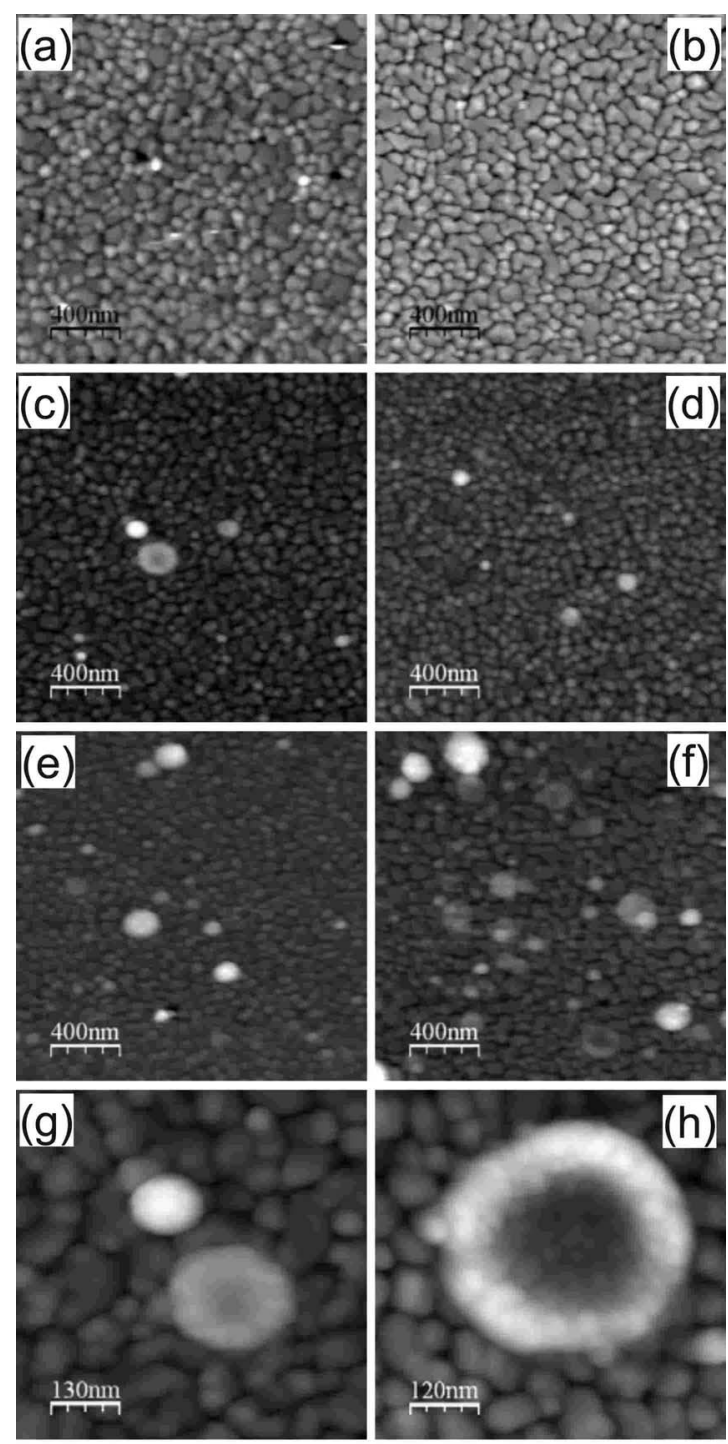

0

$Z \max$

(i)

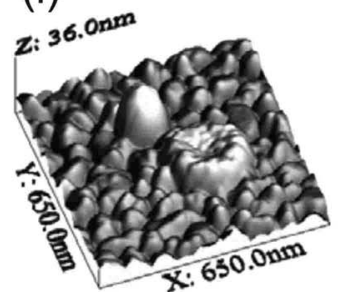

(j)

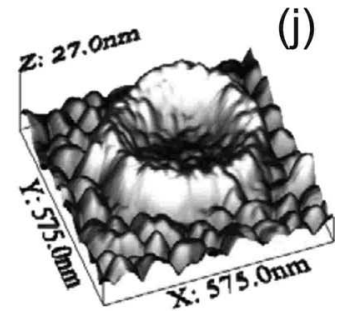

FIG. 5. AFM images for $\mathrm{Au} / 1 \mathrm{~min} \mathrm{Co} / \mathrm{Au} / \mathrm{Si}$ samples. [(a)-(f)] Representative top views of samples deposited at $0,2,3.5,7,14$, and $33 \times 10^{12} \mathrm{~W} / \mathrm{cm}^{2}$, respectively. Selected images of specific particle shapes: $[(\mathrm{g})$ and $(\mathrm{h})]$ top views and $[(\mathrm{i})$ and $(\mathrm{j})] 3 \mathrm{D}$ images of nanostructures obtained at 3.5 and $7 \times 10^{12} \mathrm{~W} / \mathrm{cm}^{2}$, respectively.

both plan-view and cross-section geometries [Figs. 6(a) and 6(b), respectively]. Both plan view and cross sections confirmed the discontinuity of the Au layer as seen by SEM. Samples in cross-section geometry were observed by EELS and HRTEM. An example corresponding to a sample grown 
(a)
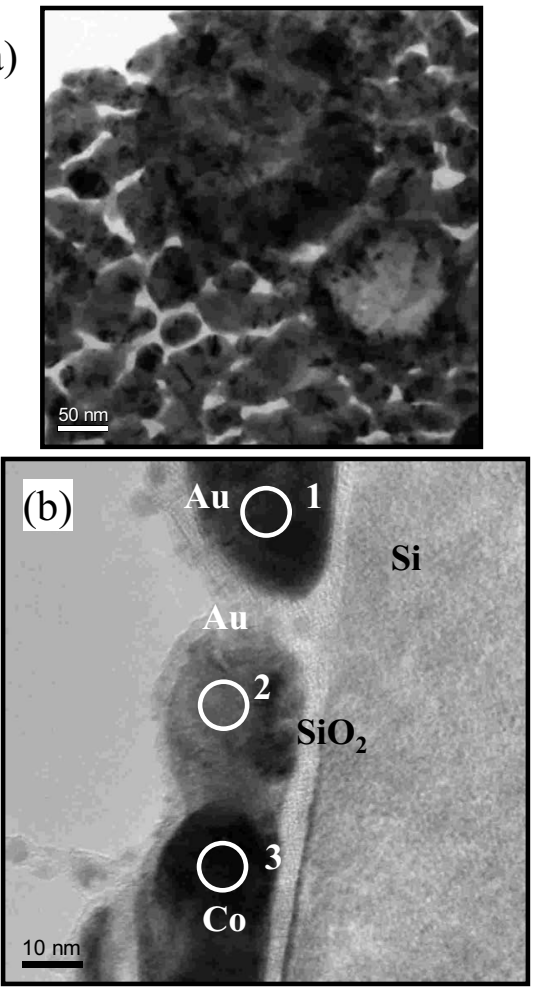

(c)

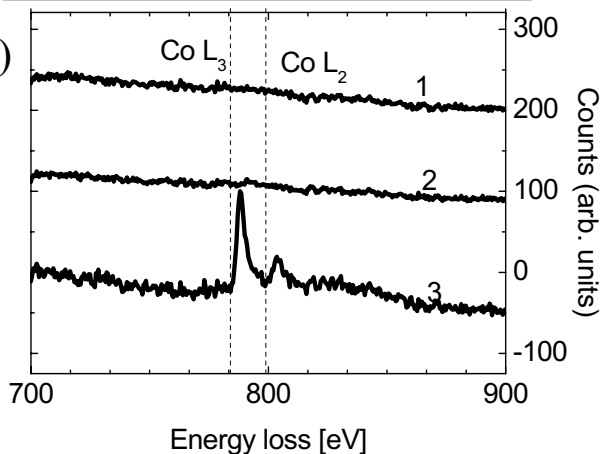

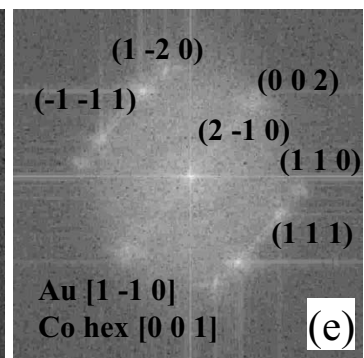

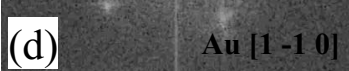

FIG. 6. (a) Plan-view image of $3.3 \times 10^{13} \mathrm{~W} / \mathrm{cm}^{2}$ sample, where Co islands can be observed. (b) Cross-section image of 1.4 $\times 10^{13} \mathrm{~W} / \mathrm{cm}^{2}$ sample, displaying a Co particle with a diameter of about $19 \mathrm{~nm}$. (c) EEL spectra in the indicated zones in (b). (d) FFT of the gold particle in (b). (e) FFT of the Co particle in (b), where $(-1-11),(002)$, and (111) reflections correspond to $\mathrm{Au}$, and $(1-20),(2-10)$, and (110) reflections correspond to hcp Co.

at $1.4 \times 10^{13} \mathrm{~W} / \mathrm{cm}^{2}$ is shown in Fig. 6(b). EELS allowed distinguishing Co particles from the Au buffer and capping discontinuous layers [Fig. 6(c)]. Within the experimental limits of the technique, no evidence of a continuous Co layer connecting the Co nanoparticles could be observed. Power

(a)
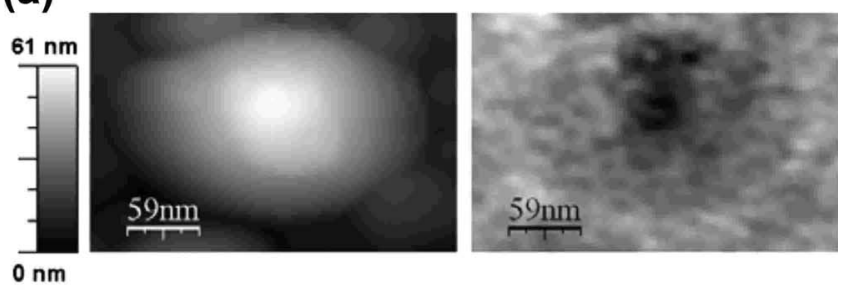

(b)
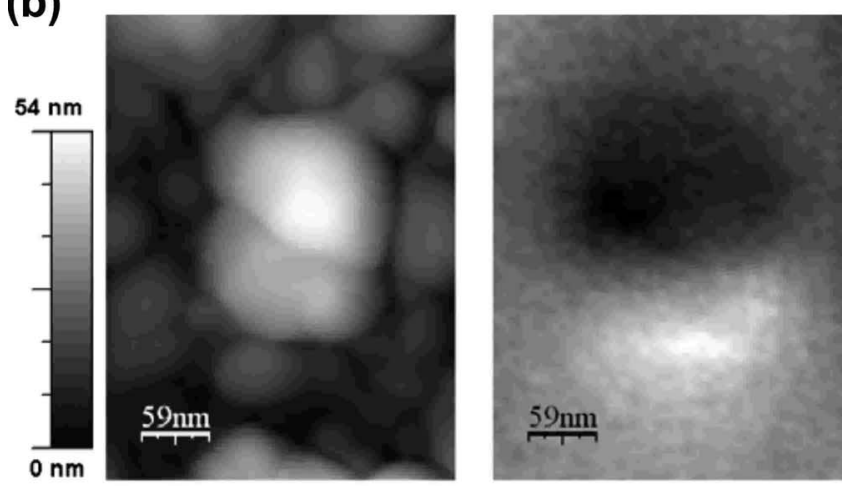

(c)

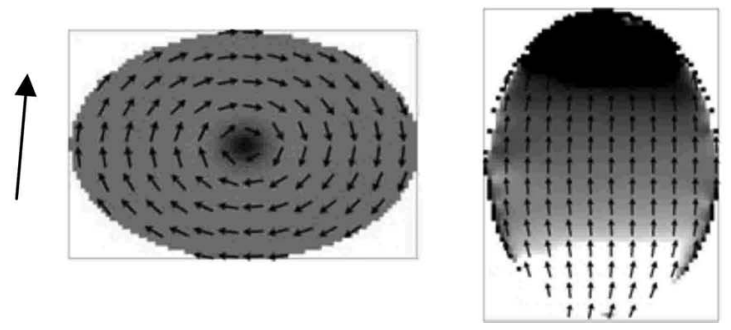

FIG. 7. [(a) and (b)] MFM images (left: topography; right: magnetic signal) for two nanoparticles exhibiting: (a) vortex state, (b) single-domain state. (c) Micromagnetic simulations for hcp nanoparticles in the remanent state after assuming a field of $2 \mathrm{~T}$ along the direction indicated by an arrow exhibiting: (left) vortex state, (right) single-domain state; the gray scale represents the magnetic charges (poles).

spectra calculated by fast Fourier transform of HRTEM images also helped to identify and discriminate between $\mathrm{Au}$ and Co particles, the latter exhibiting an hop structure [Figs. 6(d) and 6(e)]. Selected area diffraction patterns (not shown) evidenced also the presence of bright spots corresponding to $\mathrm{CoO}$.

\section{B. Magnetic properties}

\section{MFM and micromagnetic simulations}

Due to the disklike shape of the deposits, it is of interest to study the local magnetization distribution within the Co entities. This was carried out by MFM in the remanent state after in-plane saturation, although only the larger nanoparticles could be observed since the lateral resolution of MFM at ambient conditions is about $50 \mathrm{~nm}$. Two magnetic states have been found: the vortex $(\mathrm{V})$ state as shown in Fig. 7(a), and the single-domain (SD) state as shown in Fig. 7(b). The $V$ state consists of a curling spin configuration that develops a small core at the center with out-of-plane magnetization. 
Such a core creates a magnetic pole at the surface, and as MFM is a field line mapping microscopy, it gives rise to a monopolar contrast in the magnetic signal, ${ }^{38}$ i.e., the black dot at the center in the right image of Fig. 7(a). Taking into account the gray scale used in the experiments (black for attractive interaction, white for repulsive interaction), we can state that the $V$ core magnetization is parallel to the tip magnetization. Antiparallel tip and core magnetizations would have produced a white dot at the $V$ core. On the other hand, in the SD state the magnetic moments are mainly aligned along one particular in-plane direction and consequently create opposite magnetic poles at the sample ends. Such poles give rise to a dipolar contrast in the magnetic signal, i.e., black contrast at one end and white contrast at the opposite end of the nanoparticle, as shown in the right image of Fig. 7(b). The fact that the magnetic image is asymmetrical (the black end appears to be larger than the white one) has been described elsewhere and is due to the tip-sample interaction in the MFM experiment. ${ }^{39}$

It is worth mentioning that in our experiments the SD state was preferentially found in the ellipsoidal particles with the long axis not far from the direction of the initially applied magnetic field. Finally, in order to shed light on these observations, we performed micromagnetic simulations using the OOMMF $\operatorname{code}^{40}$ (parameters: exchange constant $A=3$ $\times 10^{-11} \mathrm{~J} / \mathrm{m}$, saturation magnetization $M_{s}=1.4 \times 10^{6} \mathrm{~A} / \mathrm{m}$, cell size $=3 \mathrm{~nm}$, i.e., below the exchange length for $\mathrm{Co}$ ). It is well known that the magnetically stable states exhibited by Co dots depend on the element size (lateral dimensions and thickness), crystalline anisotropy, and magnetic history. ${ }^{41,42}$ The calculations have been performed considering two ellipsoidal nanoparticles with similar thickness $(17 \mathrm{~nm})$ but different axes along the $x$ and $y$ directions: $(252 \mathrm{~nm}, 165 \mathrm{~nm})$ for one of them (named particle A) and $(168 \mathrm{~nm}, 225 \mathrm{~nm})$ for the other (particle B), mimicking the lateral dimensions of the particles shown in Figs. 7(a) and 7(b), respectively. In order to reproduce the MFM measurements, an initial inplane applied field of $2 \mathrm{~T}$ deviating $5^{\circ}$ from the $y$ axis has been considered, and the analyzed configurations correspond to the remanent states once the field is removed. The results shown in Fig. 7(c) were obtained assuming that the particles have hcp crystalline structure with magnetocrystalline easy axis always oriented along the surface normal, the anisotropy constant being $K=5.2 \times 10^{5} \mathrm{~J} / \mathrm{m}^{3}$ : particle A (on the left) exhibits a remanent $V$ state similar to that experimentally observed in Fig. 7(a), whereas particle B (on the right) exhibits a remanent SD state as that experimentally determined, as shown in Fig. 7(b). However, if an initial out-of-plane field is used instead of in-plane field, which was considered to reproduce the MFM measurements, both particles reach a remanent $V$ state. Comparing the total energy density $E$ of the two stable magnetic states obtained for particle B, i.e., SD for the initial in-plane field $\left(E=6.1 \times 10^{5} \mathrm{~J} / \mathrm{m}^{3}\right)$ or $V$ for the previous out-of-plane saturation $\left(E=5.5 \times 10^{5} \mathrm{~J} / \mathrm{m}^{3}\right)$, we can deduce that the $V$ configuration is the ground state.

From a magnetic viewpoint, it is crucial to know whether or not the magnetocrystalline easy axis has an important component along the surface normal. In order to qualitatively study the influence of this parameter on the development of the remanent state configurations after in-plane saturation, simulations varying the anisotropy constant in the perpendicular direction were performed. When the anisotropy is halved, the obtained configurations are similar to that shown in Fig. 7(c), i.e., particle A exhibits a $V$ state whereas particle $\mathrm{B}$ (on the right) exhibits an SD state. However, when the anisotropy constant is reduced by a factor of five, the remanent state for particle $\mathrm{A}$ is no longer a $V$ but a SD state with the magnetic moments mainly aligned along the long axis. This means that for the elliptical particles considered here, the development of a remanent $V$ state (where the moments at the $V$ core are pointing out of the plane) after in-plane saturation requires a global (effective) out-of-plane magnetocrystalline anisotropy high enough to overcome the shape influence. In other words, the experimental observation by MFM of remanent $V$ states points to the existence of a certain texture in the crystalline structure of the hcp particles with the $c$ axis (easy-magnetization axis) pointing out of the plane. Although the TEM measurements indicate that the nanoparticles exhibit hcp structure, the number of Co particles examined does not give significant statistics about the orientation of the easy axis.

Finally, in order to obtain an overview of the influence of the magnetocrystalline anisotropy in the development of the magnetically stable states after in-plane saturation, we performed simulations considering circular particles (in order to remove the additional shape anisotropy) with fixed thickness $(17 \mathrm{~nm})$ and different diameters. In clear contrast to the ground-state case, in which the presence of perpendicular magnetic anisotropy has little influence on the critical size for the transition from the SD ground state toward the $V$ ground state, ${ }^{43}$ we observed a strong influence of the anisotropy constant in the magnetic state obtained after in-plane saturation. In particular, for perpendicular anisotropy with $K=5.2 \times 10^{5} \mathrm{~J} / \mathrm{m}^{3}$ the critical diameter is $125 \mathrm{~nm}$ (the remanent configuration for diameter below or above such value is the $\mathrm{SD}$ state or the $V$ state, respectively), whereas for $K$ $=2.6 \times 10^{5} \mathrm{~J} / \mathrm{m}^{3}$ the critical diameter increases to $362 \mathrm{~nm}$.

\section{SQUID}

For the determination of the saturation magnetization and the possible presence of superparamagnetic nanoparticles, inplane hysteresis loops at low temperature $(10 \mathrm{~K})$ and RT were measured in all samples using a SQUID magnetometer (Fig. 8, with $m$ being the magnetic moment), confirming their ferromagnetic nature up to RT. All loops exhibit a similar shape, with no observable variation in $m_{s}$ (magnetic moment at saturation) upon reduction in temperature. The fact that the remanent moment is larger at low temperature can be easily understood by considering the configurations discussed in the previous section. Such a remanent moment is due to the particles that exhibit SD configurations (the $V$ state does not produce a net in-plane moment at zero field), and upon increasing temperature the thermal energy induces the transition to the ground state (i.e., the $V$ state) of some of them, thereby reducing the remanent moment. In Figs. 9(a) and 9(b), RT in-plane and out-of-plane hysteresis loops are shown for the $7 \times 10^{12}$ and $2.1 \times 10^{13} \mathrm{~W} / \mathrm{cm}^{2}$ samples, all of them confirming a clear in-plane magnetic anisotropy most likely produced by the morphology of the particles. The rem- 

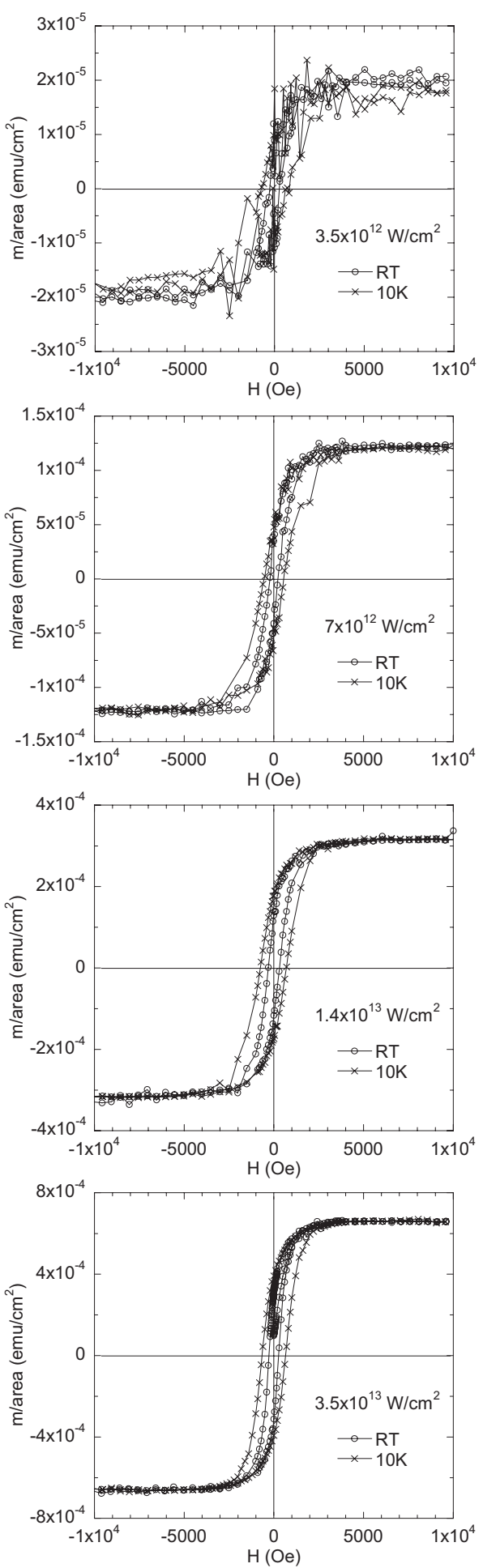

FIG. 8. Magnetic hysteresis loops vs laser power obtained from in-plane SQUID measurements at room temperature and $10 \mathrm{~K}$, showing their ferromagnetic nature up to RT.

anent moment for the out-of-plane loops can be mainly attributed to the spins at the core of the particles exhibiting $V$ states. It is worth noticing that, in spite of the minute size of the smallest detected particles, no evidence of superparamagnetism is observed in the loops. Since the measured magnetic moment could be due mainly to the larger particles, several
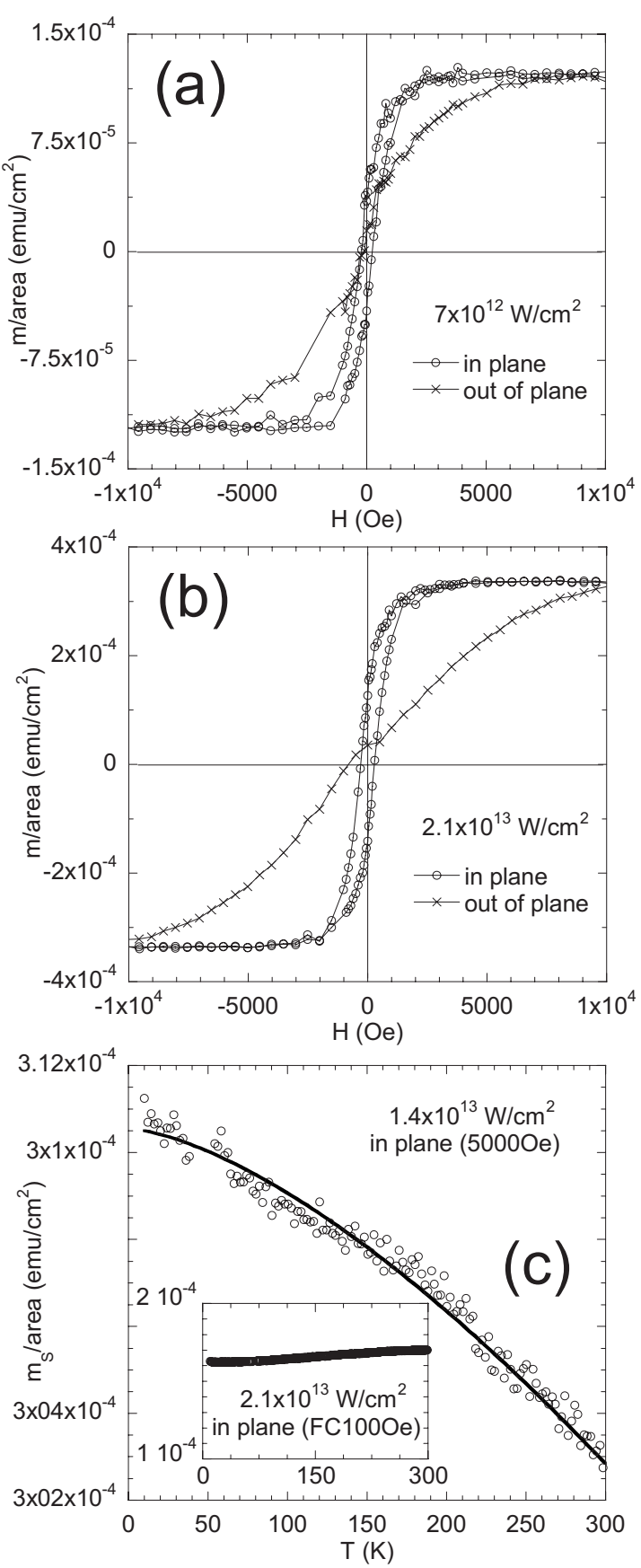

FIG. 9. [(a) and (b)] In-plane and out-of-plane magnetic hysteresis loops at RT of (a) $7 \times 10^{12} \mathrm{~W} / \mathrm{cm}^{2}$ and (b) 2.1 $\times 10^{13} \mathrm{~W} / \mathrm{cm}^{2}$ samples, showing an in-plane magnetic anisotropy. (c) $m_{S}$ vs $T$ for the $1.4 \times 10^{13} \mathrm{~W} / \mathrm{cm}^{2}$ sample, line fit corresponding to Bloch $T^{3 / 2}$ law. The inset shows the FC magnetization curve at 100 Oe for the $2.1 \times 10^{13} \mathrm{~W} / \mathrm{cm}^{2}$ sample.

temperature-dependent measurements have been performed to elucidate this point.

The $m_{s}$ vs $T$ curve is presented in Fig. 9(c) for the 1.4 $\times 10^{13} \mathrm{~W} / \mathrm{cm}^{2}$ sample, showing the Bloch $T^{3 / 2}$ law, with a $B$ factor of $4.8 \times 10^{-6}$ and with only $3 \%$ reduction in $m_{s}$ when increasing the sample temperature from $10 \mathrm{~K}$ to RT. This indicates that most of the Co aggregates are already ferromagnetic at RT, and do not exhibit any relevant amount of superparamagnetic particles and/or isolated atoms in the 


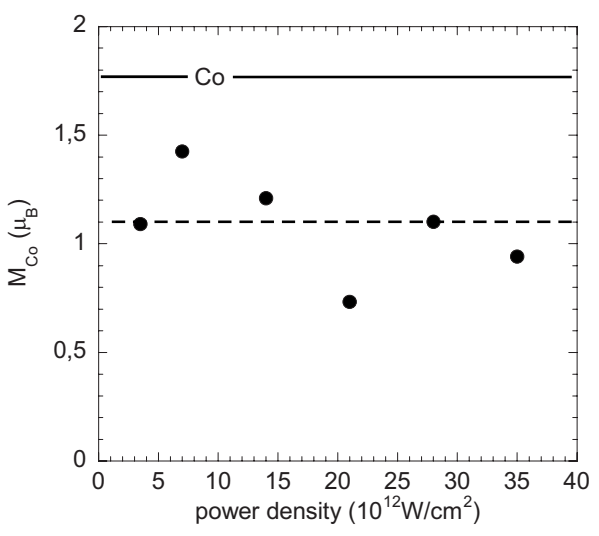

FIG. 10. The Co magnetic moment per atom estimated from the Co deposition rate, showing a low magnetic moment of $1.1 \mu_{B}$, compared to $1.77 \mu_{B}$ for bulk Co.

films. Another indication that points to the same conclusion is given in the inset of Fig. 9(c): the field-cooled (FC) magnetization curve measured with a magnetic field of $100 \mathrm{Oe}$ for the $2.1 \times 10^{13} \mathrm{~W} / \mathrm{cm}^{2}$ sample is displayed. There is no evidence for increased magnetization values at low temperatures as those expected for superparamagnetic particles and isolated ions. Only a monotonous increase in $m$ with increasing temperature is obtained, which can be understood if the assembly of ferromagnetic nanostructures was away from its equilibrium state.

Using the estimated Co deposition rate (Fig. 1) it is possible to determine the amount of $\mathrm{Co}$ in the structures and therefore the magnetic moment per Co atom as a function of the laser power (Fig. 10). In spite of the low accuracy due to both the small amount of Co in the samples and the errors in the estimation of their volume, an average value of around $1.1 \mu_{B}$ is obtained, which represents about a $40 \%$ reduction with respect to bulk Co. As will be shown in the following sections, this significant reduction in the magnetic moment can be attributed to a partial oxidation of Co.

\section{3. $X M C D$}

An independent determination of the Co magneticmoment value was carried out by XMCD measurements. Figure 11(a) displays the Co $L_{2,3}$ XAS measured for opposite magnetization directions $\left(\mu^{+}\right.$and $\left.\mu^{-}\right)$on the sample with $1.4 \times 10^{13} \mathrm{~W} / \mathrm{cm}^{2}$. A clear dependence of the absorption intensity can be observed on the magnetic polarization of the sample, as expected for ferromagnetic systems. The difference in the absorption intensities gives a direct measure of the magnetic nature of the sample at room temperature. Figure 11(b) shows the corresponding sum and difference spectra, the latter referred to as the XMCD signal, together with their corresponding integration curves obtained from a standard analysis procedure, where a double steplike function set to the peak positions of the $L_{3}$ and $L_{2}$ maxima was subtracted from the isotropic absorption spectra in order to remove the $L_{3}$ and $L_{2}$ edge jumps. The Co magnetic moments were extracted using the sum rules. ${ }^{44-46}$ Here we neglected in the analysis the so-called saturation effects, ${ }^{47,48}$ mainly because of the small thicknesses of the structures studied here. The

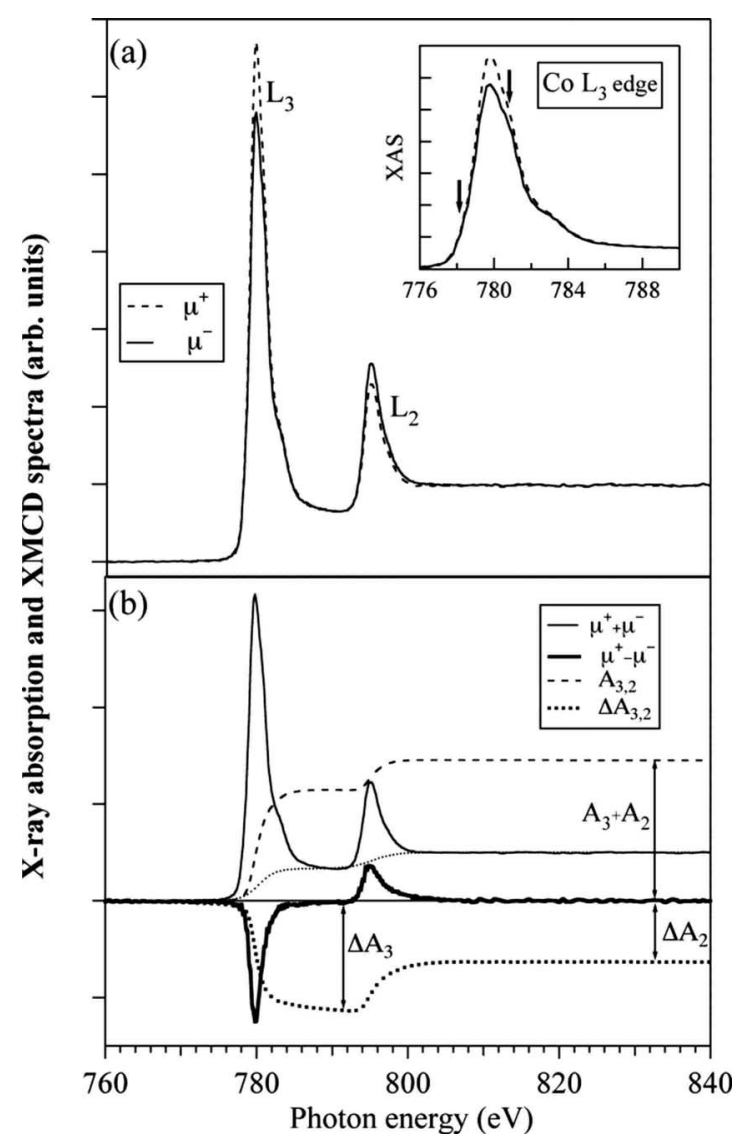

FIG. 11. (a) Normalized XAS data measured on the sample grown at $1.4 \times 10^{13} \mathrm{~W} / \mathrm{cm}^{2}$ and for opposite magnetizations. (b) Normalized summed XAS spectra, the corresponding difference spectrum (XMCD spectrum), and their integration curves. The dotted line is the two-step-like function for the edge-jump removal before the integration. The $\Delta A_{3}, \Delta A_{2}$, and $A_{3}+A_{2}$ values are the three different integrals required for the sum-rule analysis.

relation between the orbital moment $\mu_{L}$, spin moment $\mu_{S}$, magnetic-dipole term $T_{z}$, and number of $3 d$ holes, $n_{h}$ (all per atom), in the ground state and the intensities, $\Delta A_{3}, \Delta A_{2}$, and $A_{3}+A_{2}$ of the difference $\Delta A_{2,3}$ and sum $A_{2,3}$ spectra integrated over the corresponding $L_{2,3}$ edges is given by ${ }^{44-46}$

$$
\begin{gathered}
\frac{\mu_{L}}{n_{h}}=-\frac{4}{3} \frac{\Delta A_{3}+\Delta A_{2}}{A_{3}+A_{2}}, \\
\frac{\mu_{S}+7 T_{z}}{n_{h}}=-2 \frac{\Delta A_{3}-2 \Delta A_{2}}{A_{3}+A_{2}} .
\end{gathered}
$$

Theoretical results ${ }^{49}$ indicate that $7 T_{z} / \mu_{S}<0.1$, hence giving an error in $\mu_{S}$ of up to $10 \%$ by neglecting $T_{z}$.

The extracted values for the Co magnetic moments are given in Table I together with values for bulk Co taken from Ref. 46 for comparison. The values have been obtained assuming the standard value of $n_{h}=2.49$ for cobalt in the hcp structure. ${ }^{46} \mathrm{We}$ assume that the mixing of the $2 p_{3 / 2}$ and $2 p_{1 / 2}$ states as well as the contribution of the $s p$ valence band to the $L_{2,3}$ XMCD signal can be neglected and that $T_{z}=0$. 
TABLE I. Co spin and orbital atomic magnetic moments, $\mu_{L}$ and $\mu_{S}$, their ratio and the total atomic magnetic moment $\mu_{T}$ (in $\mu_{B}$ ) extracted from XMCD and XAS.

\begin{tabular}{lllll}
\hline \hline & $\begin{array}{c}\mu_{S} \\
\left(\mu_{B}\right)\end{array}$ & \multicolumn{1}{c}{$\begin{array}{c}\mu_{L} \\
\left(\mu_{B}\right)\end{array}$} & \multicolumn{1}{c}{$\mu_{L} / \mu_{S}$} & \multicolumn{1}{c}{$\begin{array}{c}\mu_{T} \\
\left(\mu_{B}\right)\end{array}$} \\
\hline Bulk Co (Ref. 46) & 1.62 & 0.154 & 0.095 & 1.77 \\
Expt. (XMCD) & $0.95(1)$ & $0.190(2)$ & $0.199(1)$ & $1.14(1)$ \\
\hline \hline
\end{tabular}

The total atomic magnetic moment obtained for this sample by XMCD is smaller than the $1.77 \mu_{B}$ reported for bulk hcp cobalt. ${ }^{46}$ The reason for this behavior could be the presence of oxidized Co. In fact, the appearance of multiplet structure [see inset of Fig. 11(a)] superimposed on the metallic Co spectrum ${ }^{50}$ strongly suggests the formation of cobalt oxide. The extra peaks in the absorption spectrum, indicated by the arrows in the inset, agree very well with those observed in the measured spectrum of $\mathrm{CoO}$ as well as with the calculated multiplet. ${ }^{51}$ Antiferromagnetic $\mathrm{CoO}$ does not contribute to the dichroic signal so that the XMCD in Fig. 11(b) only displays the metallic line shape. It is important to note here that the application of the sum rules gives the average values of the magnetic moments per atom since the denominators in Eqs. (1) and (2) include all Co atoms, i.e., metallic as well as oxidized Co atoms. Actually, the total atomic magnetic moment measured corresponded to $64 \%$ of that from bulk cobalt, in very good agreement with the values obtained with SQUID. Imperfections in the capping layer could lead to two different systems. On one hand, the system could be formed by a portion of the particles completely converted into oxide, while keeping the rest in the metallic form. On the other hand, the system could be formed by Co disks with a metallic core surrounded by an oxide shell.

The analysis of the $\mu_{L}$ and $\mu_{S}$ contributions reflected significant differences when compared to bulk cobalt. There is a strong decrease in the spin magnetic moment. Some authors reported that the high-spin orbit of gold in very small particles induces an enhancement of the $\mu_{L} / \mu_{S}$ ratio of the metal transition at the interface..$^{52,53}$ However, in the systems studied here, this effect is likely to be very attenuated as a consequence of the bigger particle size and, thus, the observed decrease in the $\mu_{S}$ would more likely be indicative of an oxidized interface. Incomplete oxidized interfaces would lead to an increase in the effective Co spin polarization $\left(\mu_{S} / n_{h}\right)$. However, once the interface is saturated with Co-O bonds, the effective Co spin polarization is reduced as more Co atoms are incorporated into the cobalt oxide ${ }^{54}$ As a consequence, the small effective Co spin polarization measured by XMCD suggests the formation of a continuous paramagnetic cobalt oxide at the Co interface.

On the other hand, an increase in the orbital magnetic moment was measured compared to bulk cobalt. For the nanoparticles the orbital to spin magnetic-moment ratio is roughly doubled (see Table I). This result is in agreement with earlier observations on Co clusters that found a strong variation in the atomic magnetic moment with cluster size. ${ }^{55-57}$ The orbital magnetic moment is directly linked to the electronic structure and its size depends on the $3 d$ hy-

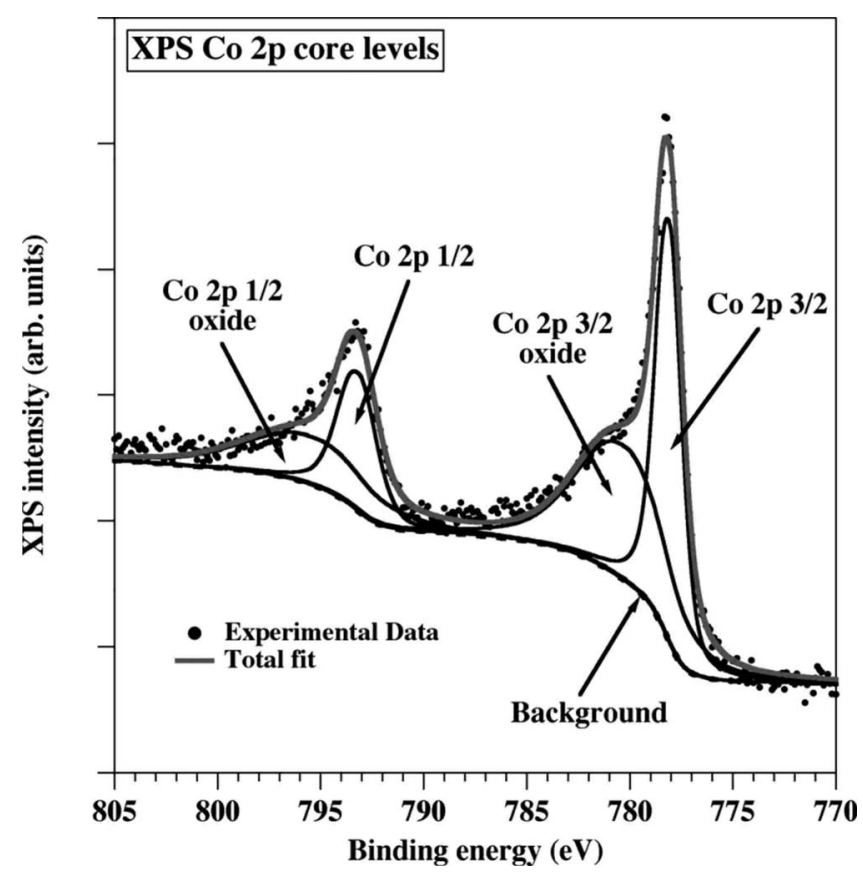

FIG. 12. Representative Co $2 p$ core-level XPS spectrum of the sample grown at $1.4 \times 10^{13} \mathrm{~W} / \mathrm{cm}^{2}$.

bridization and localization. The increase is generally ascribed to electron localization at the perimeter of the clusters. The orbital magnetic moment is very sensitive to small changes in the surface structure ${ }^{58}$ and strongly depends on the geometry of the particle. ${ }^{59} \mathrm{An}$ enhancement also occurs if the Co is at a site where its orbital moment is not quenched by the crystal field, which for instance is the case for $\mathrm{Co}^{2+}$ in octahedral symmetry, such as for $\mathrm{CoO} .{ }^{51}$ However, to observe this in the XMCD spectrum would require magnetic moments that are not antiferromagnetically coupled.

\section{Chemical characterization: XPS}

A very likely reason for the observed reduced magnetic moment with respect to that of the bulk is the oxidation of Co due to the discontinuous nature of the Au capping layers, and therefore their effective role against oxidation of Co was directly checked by XPS. A representative Co $2 p$ core-level spectrum is displayed in Fig. 12. Together with the experimental data points, the background and the fitting curves are displayed. As can be observed, the experimental spectrum has been fitted with four components that correspond to the Co $2 p_{1 / 2}$ and $2 p_{3 / 2}$ core levels and their corresponding $\mathrm{Co}$ oxide components. From the integrated area of the components of the Co core levels and from the photoemission peaks of gold and oxygen (not shown here) and taking into account the corresponding photoemission cross sections, the proportion of Co oxide compared to the metallic Co was found to be $\sim 50 \%$. More precisely, the chemical composition extracted from the XPS data is presented in Table II. The proportion of cobalt oxides and pure metallic cobalt found by XPS $(\sim 50 \%)$ is in good agreement with the reduction in the total atomic magnetic moment determined by XMCD ( $\sim 36 \%$ as compared to the atomic magnetic moment of pure 
TABLE II. Chemical composition as deduced from the XPS spectra (at. \%) from representative sample.

\begin{tabular}{|c|c|c|c|c|c|c|}
\hline $\mathrm{Au} 4 f_{5 / 2}$ & $\mathrm{Au} 4 f_{7 / 2}$ & $\mathrm{O} 1 s$ & Co $2 p_{1 / 2}$ & Co $2 p_{3 / 2}$ & $\begin{array}{c}\text { Co } 2 p_{1 / 2} \\
\text { oxide }\end{array}$ & $\begin{array}{c}\text { Co } 2 p_{3 / 2} \\
\text { oxide }\end{array}$ \\
\hline 38 & 38 & 14 & 2.3 & 2.2 & 2.0 & 2.3 \\
\hline \multicolumn{2}{|c|}{76} & 14 & \multicolumn{2}{|c|}{4.5} & \multicolumn{2}{|c|}{4.3} \\
\hline
\end{tabular}

metallic hcp Co) and SQUID ( $40 \%$ as compared to the atomic magnetic moment of pure metallic hcp Co). Such results clearly evidence the noncontinuity of the Au capping layer that induces a partial oxidation of the Co particles and in turn a reduction in the effective magnetic moment or magnetization.

Finally, it is worth mentioning that the existence of antiferromagnetic $\mathrm{CoO}$ usually induces unidirectional anisotropy in Co particle systems. ${ }^{60}$ However, in our samples no shift in the hysteresis loops was observed at low temperature after field cooling, i.e., no exchange bias has been detected. It is very likely that the oxide is irregular and nonstoichiometric so the amount of antiferromagnetically ordered $\mathrm{CoO}$ is small and not able to produce exchange bias in the structures.

\section{DISCUSSION}

Focusing first on the structural and morphological aspects, we systematically observed the formation of nanoparticles for all ablation powers above the ablation threshold, with an expected increase in the amount of deposited material with laser power. The particle size distribution does not show any noticeable differences when the laser power is increased, except for the observation of larger particles at larger powers. An important aspect is the absence of a characteristic peak for a specific particle size in the size distribution, with instead a continuously increasing number of particles for smaller diameters. This result, in contrast with the findings of other authors reporting a characteristic particle size, is in agreement with theoretical calculations that describe the ablation process as the emission of particles with a wide range of sizes, all the way down to the atomic size. Comparing with ablation techniques that use longer laser pulses, namely ns-PLD, where the presence of a high-pressure gas is necessary to obtain nanoparticles ${ }^{61,62}$ in our specific case nanoparticles are spontaneously obtained from the ablation process. The specific research reported here on the properties of nanoparticle systems provides a technically easier fabrication method of the desired structures. From morphological viewpoint, the obtained structures have systematically a disklike, instead of a spherelike shape, indicative of a liquid or at least plastic state of the particle impinging on the substrate, being deformed to this final shape. It is noticeable that, in addition to flat circular disks, in some cases other morphologies are also observed, such as rings and rosaries, which indicate a matter redistribution of the particle upon arrival at the substrate surface. This is of special interest in the case of magnetic materials since these shapes could potentially give rise to magnetic domain structures and anisotropies characteristic of these morphologies.
Two series of samples were grown, one directly on Si and the other one on a Au buffer layer on glass and subsequently covered with $\mathrm{Au}$. The morphology of the Au buffer layer is not flat over large lateral dimensions, but is characterized by a granular nature due to the poor wetting of $\mathrm{Au}$ on glass. However, the final morphology of the particles in the $\mathrm{Au} / \mathrm{Co} / \mathrm{Au}$ structures is very similar to the $\mathrm{Co} / \mathrm{Si}$ ones, indicating first that the Au capping layer must be substantially conformal with the Co structures underneath, reproducing their shapes; and second that the morphology of the Co structures does not strongly depend on whether these are deposited on $\mathrm{Si}$ (or actually its native oxide) or Au. This last point indicates that the final obtained morphologies are mainly determined by the state of the Co particles before arrival at the substrate, probably because these are in a liquid or at least a plastic state with a kinetic energy sufficient to give rise to particle deformation which produces the final observed shapes.

From magnetic properties viewpoint, only studied in the $\mathrm{Au} / \mathrm{Co} / \mathrm{Au}$ samples, we obtain a reduced magnetic moment per atom basically independent of the laser power. Within a rather wide range of laser power values, varying between 1.4 and $0.7 \mu_{B}$ while the bulk value is $1.77 \mu_{B}$. This result, confirmed by both SQUID and XMCD measurements, is due to the partial Co oxidation probably caused by a poor coverage of the Au capping layer. Both SQUID and XMCD are experimental techniques that probe large lateral areas, and therefore, even though the studied specimens present a wide range of particle sizes, the obtained results correspond to an average over the whole macroscopic system. In this respect, we have also applied a microscopical magnetic characterization technique such as MFM, which allows obtaining the domain structures of some of the observed morphologies, which are well reproduced by micromagnetic simulations. Moreover, these magnetic states can explain the macroscopic hysteresis loops measured by SQUID, both for in-plane and out-of-plane fields. On the other hand, we do not find any experimental evidence for superparamagnetism in the SQUID measurements. This is consistent with the smallest particle size detected in our SEM and AFM measurements, but it would be a surprising result if even smaller Co particles were present.

It is obvious that, for fs-PLD to become of relevance in a near future as a standard deposition technique for the fabrication of magnetic nanostructures, many aspects need to be improved, especially concerning the particle size distribution. However, we hope that this work will contribute to a deeper understanding of the morphological, structural, and magnetic properties of magnetic systems obtained by fsPLD. 


\section{CONCLUSIONS}

A comprehensive study covering structural, morphological, magnetic, and chemical characterization has been performed in Co structures grown by fs-PLD. The potential of fs-PLD as a powerful technique for the fabrication of magnetic entities of nanometric dimensions over large areas has been also demonstrated. Usually, nanodisks are obtainedtheir maximum size as well as the particle number increasing with the laser power density. Particles covered with Au were studied magnetically, showing no indication of superparamagnetism in spite of the small size of many of them. The measured magnetization exhibits some evolution with the laser power; however, the uncertainty in the determination of the actual amount of $\mathrm{Co}$ and the partial oxidation due to the discontinuous Au layer deposited on top make further studies necessary to clarify this issue.

Two remanent states have been identified after in-plane saturation: vortex and single-domain states, with the global magnetism dominated by the monodomain particles. We want to emphasize that the noncontinuity of the Au capping layer that induces the partial oxidation of the Co particles is an important aspect of the findings and contrasts with the behavior obtained in structures fabricated by, for example, MBE or sputtering. It highlights the different nature of the fs-PLD grown Co nanoentities with respect to those obtained by other physical vacuum deposition methods

The magnetic properties found in these structures are consistent with the presence of such a partial oxidation. The reduction in the effective magnetic volume explains the reduction in magnetic moment with respect to the bulk. The nonevidence of superparamagnetism is not only due to the disklike shape of the particles that increases the effective anisotropy, but is probably also related to the fact that the smallest particles are completely oxidized and consequently became nonmagnetic.

\section{ACKNOWLEDGMENTS}

Work was supported in part by the U.S. Department of Energy, Basic Energy Sciences (Grant No. DE-FG0206ER46273), NSF FOCUS Center, the Spanish Ministerio de Educación y Ciencia (References No. PR2005-0017 and No. MAT2005-05524-C02), Comunidad de Madrid (Reference No. S-0505/MAT/0194 NANOMAGNET), and CSIC (Reference No. 200650I130). Support from the SRS staff during the XMCD experiments is greatly acknowledged. Y.H. and L.M. also acknowledge financial support from the "Ramón y Cajal" and "Juan de la Cierva" programs, respectively, from the Spanish Ministerio de Investigación y Ciencia and Consejo Superior de Investigaciones Científicas (CSIC).
${ }^{1}$ F. Vidal, T. W. Johnston, S. Laville, O. Barthélemy, M. Chaker, B. Le Drogoff, J. Margot, and M. Sabsabi, Phys. Rev. Lett. 86, 2573 (2001).

${ }^{2}$ D. Perez and L. J. Lewis, Phys. Rev. Lett. 89, 255504 (2002).

${ }^{3}$ T. E. Glover, J. Opt. Soc. Am. B 20, 125 (2003).

${ }^{4}$ D. Perez and L. J. Lewis, Phys. Rev. B 67, 184102 (2003).

${ }^{5}$ P. Lorazo, L. J. Lewis, and M. Meunier, Phys. Rev. Lett. 91, 225502 (2003).

${ }^{6}$ S. D. Brorson, J. G. Fujimoto, and E. P. Ippen, Phys. Rev. Lett. 59, 1962 (1987).

${ }^{7}$ K. Sokolowski-Tinten, J. Bialkowski, A. Cavalleri, D. von der Linde, A. Oparin, J. Meyer-ter-Vehn, and S. I. Anisimov, Phys. Rev. Lett. 81, 224 (1998).

${ }^{8}$ A. Cavalleri, K. Sokolowski-Tinten, J. Bialkowski, M. Schreiner, and D. von der Linde, J. Appl. Phys. 85, 3301 (1999).

${ }^{9}$ D. von der Linde and K. Sokolowski-Tinten, Appl. Surf. Sci. 154-155, 1 (2000).

${ }^{10}$ N. N. Nedialkov, P. A. Atanasov, S. E. Imamova, A. Ruf, P. Berger, and F. Dausinger, Appl. Phys. A: Mater. Sci. Process. 79, 1121 (2004).

${ }^{11}$ F. Korte, J. Koch, and B. N. Chichkov, Appl. Phys. A: Mater. Sci. Process. 79, 879 (2004).

${ }^{12}$ B. Rethfeld, K. Sokolowski-Tinten, D. von der Linde, and S. I. Anisimov, Appl. Phys. A: Mater. Sci. Process. 79, 767 (2004).

${ }^{13}$ M. Vitiello, S. Amoruso, C. Altucci, C. de Lisio, and X. Wang, Appl. Surf. Sci. 248, 163 (2005).

${ }^{14}$ H. Dachraoui and W. Husinsky, Appl. Phys. Lett. 89, 104102 (2006).

${ }^{15}$ H.-Y. Lai, P.-H. Huang, and T.-H. Fang, Appl. Phys. A: Mater. Sci. Process. 86, 497 (2007).
${ }^{16}$ E. Millon, O. Albert, and J. C. Loulergue, J. Appl. Phys. 88, 6937 (2000).

${ }^{17}$ Z. Zhang, P. A. VanRompay, J. A. Nees, R. Clarke, X. Pan, and P. P. Pronko, Appl. Surf. Sci. 154, 165 (2000).

${ }^{18}$ J. E. Dominguez, L. Fu, and X. Q. Pan, Appl. Phys. Lett. 79, 614 (2001).

${ }^{19}$ J. E. Dominguez, X. Q. Pan, L. Fu, P. A. Van Rompay, Z. Zhang, J. A. Nees, and P. P. Pronko, J. Appl. Phys. 91, 1060 (2002).

${ }^{20}$ J. Perrière, E. Millon, W. Seiler, and C. Boulmer-Leborgne, J. Appl. Phys. 91, 690 (2002).

${ }^{21}$ D. Brodoceanu, A. Manousaki, I. Zergioti, A. Klini, M. Dinescu, and C. Fotakis, Appl. Phys. A: Mater. Sci. Process. 79, 911 (2004).

${ }^{22}$ P. P. Pronko, Z. Zhang, and P. A. VanRompay, Appl. Surf. Sci. 208, 492 (2003)

${ }^{23}$ A. Reilly, C. Allmond, S. Watson, J. Gammon, and J. Gi Kim, J. Appl. Phys. 93, 3098 (2003).

${ }^{24}$ S. Amoruso, R. Bruzzese, N. Spinelli, R. Velotta, M. Vitiello, X. Wang, G. Ausanio, V. Iannotti, and L. Lanotte, Appl. Phys. Lett. 84, 4502 (2004).

${ }^{25}$ S. Amoruso, R. Bruzzese, N. Spinelli, R. Velotta, M. Vitiello, and X. Wang, Europhys. Lett. 67, 404 (2004).

${ }^{26}$ O. Albert, S. Roger, Y. Glinec, J. C. Loulergue, J. Etchepare, C. Boulmer-Leborgne, J. Perrière, and E. Millon, Appl. Phys. A: Mater. Sci. Process. 76, 319 (2003).

${ }^{27}$ S. Eliezer, N. Eliaz, E. Grossman, D. Fisher, I. Gouzman, Z. Henis, S. Pecker, Y. Horovitz, M. Fraenkel, S. Maman, and Y. Lereah, Phys. Rev. B 69, 144119 (2004)

${ }^{28}$ G. Ausanio, A. C. Barone, V. Iannotti, L. Lanotte, S. Amoruso, R. Bruzzese, and M. Vitiello, Appl. Phys. Lett. 85, 4103 (2004). 
${ }^{29}$ B. Liu, Z. Hu, Y. Che, Y. Chen, and X. Pan, Appl. Phys. Lett. 90, 044103 (2007).

${ }^{30}$ S. Amoruso, G. Ausanio, R. Bruzzese, L. Lanotte, P. Scardi, M. Vitiello, and X. Wang, J. Phys.: Condens. Matter 18, L49 (2006).

${ }^{31}$ S. Amoruso, G. Ausanio, R. Bruzzese, M. Vitiello, and X. Wang, Phys. Rev. B 71, 033406 (2005).

${ }^{32}$ C. Hermann, V. A. Kosobukin, G. Lampel, J. Peretti, V. I. Safarov, and P. Bertrand, Phys. Rev. B 64, 235422 (2001).

${ }^{33}$ V. I. Safarov, V. A. Kosobukin, C. Hermann, G. Lampel, J. Peretti, and C. Marlière, Phys. Rev. Lett. 73, 3584 (1994).

${ }^{34}$ J. B. González-Díaz, A. García-Martín, G. Armelles, J. M. García-Martín, C. Clavero, A. Cebollada, R. A. Lukaszew, J. R. Skuza, D. P. Kumah, and R. Clarke, Phys. Rev. B 76, 153402 (2007).

${ }^{35}$ J. B. González-Díaz, A. García-Martín, J. M. García-Martín, A. Cebollada, G. Armelles, B. Sepúlveda, Y. Alaverdyan, and M. Käll, Small 4, 202 (2008).

${ }^{36}$ M. S. Martín-González, Y. Huttel, A. Cebollada, G. Armelles, and F. Briones, Surf. Sci. 571, 63 (2004).

${ }^{37}$ D. Kumah, A. Cebollada, C. Clavero, J. M. García-Martín, J. R. Skuza, R. A. Lukaszew, and R. Clarke, J. Phys. D 40, 2699 (2007).

${ }^{38}$ J. M. García-Martín, A. Thiaville, J. Miltat, T. Okuno, L. Vila, and L. Piraux, J. Phys. D 37, 965 (2004).

${ }^{39}$ J. M. García, A. Thiaville, and J. Miltat, J. Magn. Magn. Mater. 249, 163 (2002).

${ }^{40}$ M. J. Donahue and D. G. Porter, OOMmF User's Guide, National Institute of Standards and Technology Interagency Report No. NISTIR 6376 (unpublished); this public code can be found at http://math.nist.gov/oommf/

${ }^{41}$ M. Hehn, K. Ounadjela, J.-P. Bucher, F. Rousseaux, D. Decanini, B. Bertenlian, and C. Chappert, Science 272, 1782 (1996).

${ }^{42}$ L. D. Buda, I. L. Prejbeanu, U. Ebels, and K. Ounadjela, Comput. Mater. Sci. 24, 181 (2002).

${ }^{43}$ L. D. Buda, I. L. Prejbeanu, M. Demand, U. Ebels, and K. Ounadjela, IEEE Trans. Magn. 37, 2061 (2001).

${ }^{44}$ B. T. Thole, P. Carra, F. Sette, and G. van der Laan, Phys. Rev. Lett. 68, 1943 (1992).

${ }^{45}$ P. Carra, B. T. Thole, M. Altarelli, and X. Wang, Phys. Rev. Lett. 70, 694 (1993).

${ }^{46}$ C. T. Chen, Y. U. Idzerda, H.-J. Lin, N. V. Smith, G. Meigs, E.
Chaban, G. H. Ho, E. Pellegrin, and F. Sette, Phys. Rev. Lett. 75, 152 (1995).

${ }^{47}$ R. Nakajima, Ph.D. thesis, Stanford University, 1998.

${ }^{48}$ R. Nakajima, J. Stöhr, and Y. U. Idzerda, Phys. Rev. B 59, 6421 (1999).

${ }^{49}$ A. Scherz, H. Wende, K. Baberschke, J. Minár, D. Benea, and H. Ebert, Phys. Rev. B 66, 184401 (2002).

${ }^{50}$ N. D. Telling, G. van der Laan, S. Ladar, R. J. Hicken, and E. Arenholz, J. Appl. Phys. 99, 08E505 (2006).

${ }^{51}$ G. van der Laan, E. Arenholz, R. V. Chopdekar, and Y. Suzuki, Phys. Rev. B 77, 064407 (2008).

${ }^{52}$ J. Bartolomé, L. M. García, F. Bartolomé, F. Luis, R. LópezRuiz, F. Petroff, C. Deranlot, F. Wilhelm, A. Rogalev, P. Bencok, N. B. Brookes, L. Ruiz, and J. M. González-Calbet, Phys. Rev. B 77, 184420 (2008).

${ }^{53}$ O. Sipr, S. Bornemann, J. Minár, S. Polesya, V. Popescu, A. Simůnek, and H. Ebert, J. Phys.: Condens. Matter 19, 096203 (2007).

${ }^{54}$ N. D. Telling, G. van der Laan, S. Ladar, and R. J. Hicken, Appl. Phys. Lett. 85, 3803 (2004).

${ }^{55}$ H. A. Durr, S. S. Dhesi, E. Dudzik, D. Knabben, G. van der Laan, J. B. Goedkoop, and F. U. Hillebrecht, Phys. Rev. B 59, R701 (1999).

${ }^{56}$ E. Dudzik, H. A. Dürr, S. S. Dhesi, G. van der Laan, D. Knabben, and J. B. Goedkoop, J. Phys.: Condens. Matter 11, 8445 (1999).

${ }^{57}$ K. W. Edmonds, C. Binns, S. H. Baker, S. C. Thornton, C. Norris, J. B. Goedkoop, M. Finazzi, and N. B. Brookes, Phys. Rev. B 60, 472 (1999).

${ }^{58}$ F. May, M. Tischer, D. Arvanitis, M. Russo, J. H. Dunn, H. Henneken, H. Wende, R. Chauvistré, N. Mårtensson, and K. Baberschke, Phys. Rev. B 53, 1076 (1996).

${ }^{59}$ R. A. Guirado-López and J. M. Montejano-Carrizales, Phys. Rev. B 75, 184435 (2007).

${ }^{60}$ W. H. Meiklejohn and C. P. Bean, Phys. Rev. 105, 904 (1957).

${ }^{61}$ J. M. González, M. I. Montero, L. Vázquez, J. A. Martín Gago, D. Givord, C. de Julián, and K. O’Grady, J. Magn. Magn. Mater. 196, 96 (1999).

${ }^{62}$ Happy, S. R. Mohanty, P. Lee, T. L. Tan, S. V. Springham, A. Patran, R. V. Ramanujan, and R. S. Rawat, Appl. Surf. Sci. 252, 2806 (2006). 